\title{
BMJ Open First clinical postmarketing experiences in the treatment of epilepsies with brivaracetam: a retrospective observational multicentre study
}

\author{
Katja Menzler (D) , ${ }^{1}$ Peter Michael Mross, ${ }^{1}$ Felix Rosenow, ${ }^{1,2,3}$ \\ Susanne Schubert-Bast (D) ,,3,4 Laurent Maximilian Willems (D) , ${ }^{2}$ Felix Zahnert, ${ }^{1}$ \\ Ilka Immisch, ${ }^{1}$ Sven Fuest, ${ }^{1}$ Felix von Podewils, ${ }^{5}$ Rhina Kunz, ${ }^{5}$ Martin Hirsch, ${ }^{6}$ \\ Tamara Mueller, ${ }^{7}$ Justus Marquetand, ${ }^{8}$ Yaroslav Winter, ${ }^{9}$ Lisa Langenbruch, ${ }^{10}$ \\ Michal Cicanic, ${ }^{11}$ Stefan Beyenburg, ${ }^{12}$ Adam Strzelczyk, ${ }^{1,2,3}$ Susanne Knake ${ }^{1,3}$
}

To cite: Menzler K, Mross PM, Rosenow $\mathrm{F}$, et al. First clinical postmarketing experiences in the treatment of epilepsies with brivaracetam: a retrospective observational multicentre study. BMJ Open 2019;9:e030746. doi:10.1136/ bmjopen-2019-030746

- Prepublication history for this paper is available online. To view these files, please visit the journal online (http://dx.doi. org/10.1136/bmjopen-2019030746).

Received 09 April 2019 Revised 20 September 2019 Accepted 26 September 2019

Check for updates

(c) Author(s) (or their employer(s)) 2019. Re-use permitted under CC BY-NC. No commercial re-use. See rights and permissions. Published by BMJ.

For numbered affiliations see end of article.

Correspondence to

Dr Katja Menzler;

hattemer@med.uni-marburg.de

\section{ABSTRACT}

Objectives Brivaracetam (BRV) is the latest approved antiepileptic drug and acts as a synaptic vesicle protein $2 \mathrm{~A}$ ligand. The aim of the present study was to evaluate the efficacy and tolerability of BRV in the clinical setting. Design Retrospective, observational multicentre study. Setting We retrospectively collected clinical data of patients who received BRV in 10 epilepsy centres using a questionnaire that was answered by the reporting neurologist.

Participants Data of 615 epilepsy patients treated with BRV were included in the study.

Primary and secondary outcome measures Efficacy regarding seizure frequency and tolerability of BRV were evaluated. Descriptive statistics complemented by $X^{2}$ contingency tests and effect sizes were performed.

Results Overall, $44 \%$ of the patients had a decreased, $38 \%$ a stable and $18 \%$ an increased seizure frequency. $17 \%$ of patients achieved seizure freedom after initiation of BRV. The seizure frequency decreased in $63 \%$ of 19 patients with BRV monotherapy. $27 \%$ reported adverse effects, but only $10 \%$ of patients with monotherapy. Brivaracetam was significantly more often associated with decreased seizure frequency in levetiracetam (LEV) naïve patients ( $p=0.012)$, but BRV also led to a decreased seizure frequency in $42 \%$ of patients who had been treated with LEV before, including $17 \%$ of patients who were completely seizure free. Adverse effects under LEV improved in $62 \%$ and deteriorated in $2 \%$ of patients after the switch to BRV. At latest follow-up (mean \pm SD = $26.3 \pm 6.5$ months), $68 \%$ were still on BRV.

Conclusions The present study shows that results of the phase III studies on BRV match data from real life clinical settings. Brivaracetam seems to be a useful alternative in patients who have suffered adverse effects while taking LEV.

\section{INTRODUCTION}

Epilepsy is a common neurological disorder, affecting about $0.7 \%$ of the general population. ${ }^{1}$ Up to $30 \%$ of epilepsy patients continue

\section{Strengths and limitations of this study}

- This study extends our knowledge about the efficacy and tolerability of brivaracetam (BRV) beyond phase III studies into real life clinical settings.

- The inclusion of patients of 10 epilepsy centres results in a large number of 615 patients.

- The study provides data on the efficacy and tolerability of BRV as off-label monotherapy, where sufficient clinical data are lacking so far.

Due to the real life clinical setting and the retrospective analysis, data were not collected in a systematic way, for example, seizure frequency and side effects rely on the patient history, comedication was not kept stable and the follow-up period was variable.

- These limitations in the study design only allow for descriptive statistics and very limited statistical tests, as more elaborate statistical analyses would overstress the data.

to have seizures despite adequate medication with currently available antiepileptic drugs (AED). ${ }^{2}$ The high prevalence of pharmacoresistant epilepsy exposes the need to develop new AED. Also, AED with minimal side effects are required, as tolerability is the most important factor influencing adherence. ${ }^{34}$

Brivaracetam (BRV) is a new AED approved in patients aged 16 or above for the add-on treatment of focal-onset seizures, with or without secondary generalisation. ${ }^{5}$ Like levetiracetam (LEV), brivaracetam acts as a synaptic vesicle protein 2A ligand with a 15 to 30 times higher binding potential than LEV. ${ }^{6}$

A pooled analysis of three phase III studies revealed a reduction in seizure frequency of $18.5 \%$ (50 mg/day), $24.4 \%$ (100 mg/day) and $24.0 \%$ (200 mg/day) over placebo and a $\geq 50 \%$ responder rate of $43.2 \%$ (50 mg/day), 
$39.5 \%$ (100 mg/day) and 37.8\% (200 mg/day). Among patients treated with BRV, $68.0 \%$ reported adverse events (AE) as compared with $62.1 \%$ of patients taking placebo, mainly somnolence, dizziness, headache and fatigue. ${ }^{6}$

The aim of the present study was to evaluate the effectiveness and tolerability of BRV in the postmarketing period to evaluate if the reduction in seizure frequency and adverse events reported in the phase III studies can be extended into the clinical setting. Also, the proportion of patients still on BRV at latest follow-up was calculated.

\section{METHODS}

In this retrospective, observational multicentre study, data from epilepsy patients who received BRV anytime between February 2016 (approval of this AED in Germany) and October 2018 and had at least one follow-up visit were collected in 10 different epilepsy centres in Germany and Luxemburg. All German speaking epilepsy centres were asked for participation via email using the monthly letter from the German International League Against Epilepsy chapter.

We used a questionnaire that was answered by the reporting neurologist using medical records of the patients. We collected baseline data on age, sex, duration of epilepsy, aetiology, seizure frequency, dose and titration of BRV, current treatment with other AED, as well as psychiatric comedication. Due to the similar modes of action, previous treatment with LEV and side effects during treatment with LEV were also recorded.

At each site, we also collected follow-up data. We documented the time period between initiation of BRV and the latest follow-up while still on BRV. A minimum follow-up period of 3 months was required. The recorded data at follow-up included seizure frequency, $\mathrm{AE}$, possible changes of adverse events during previous treatment with LEV and discontinuation of BRV together with the corresponding reasons.

Regarding seizure outcome, the seizure frequency was determined from the medical history of the patients at the time of introduction of BRV and latest follow-up while still on treatment. Information on seizure frequency was considered unreliable if information on seizure frequency was not documented in the patients' charts or patients could not report the number of seizures. If information on seizure frequency was missing, patients were excluded from the analysis of this parameter, but these patients were still included in the analysis of adverse events or proportion of patients still on BRV. The follow-up period was variable and the seizure frequencies reported ranged from less than once a month to more than 1000 a month. Also, in several patient charts a range of seizure frequencies like four to eight seizures per month was documented. We therefore decided to only report broad categories including complete seizure freedom and stable, increased or decreased seizure frequency. Any changes in seizure frequency were interpreted as increase or decrease, no minimum percentage of increase or decrease was required. In our opinion any more detailed analysis would suggest an accuracy that is not in the data.

Changes in seizure frequency and adverse events as well as the proportion of patients still taking BRV at latest follow-up were evaluated in the whole group of patients. In addition, subgroups of LEV naïve patients and patients who had been treated with LEV before were analysed separately due to the similar mode of action of BRV and LEV that might influence the efficacy or tolerability. Also, a subgroup of patients with off-label use of BRV in monotherapy was analysed separately.

Statistical analysis was performed using the Statistical Package for Social Science (SPSS, IBM) V.24. The analysis focused primarily on descriptive statistics that were complemented by $\mathrm{X}^{2}$ contingency tests and effect sizes (Cramer's V).

In this manuscript we followed the Strengthening the Reporting of Observational Studies in Epidemiology guidelines for the reporting of observational studies.

\section{Patient and public involvement statement}

Patients were not involved in this retrospective data analysis.

\section{RESULTS}

\section{Patient characteristics}

Data from 615 patients were collected in 10 epilepsy centres. Three hundred and four patients (49\%) were male, 311 patients $(51 \%)$ were female. The mean age was $40.8 \pm 17.0($ mean \pm SD) years.

Information on epilepsy duration was available in 589 patients. The mean duration of epilepsy was 21.0 \pm 14.6 $($ mean $\pm \mathrm{SD})$ years. The median seizure frequency of 600 patients was 5 (range 0 to 1000) per month. The sample included 25 patients with genetic generalised epilepsy.

Sixty patients $(10 \%)$ had psychiatric medication as an indicator of psychiatric comorbidity.

The mean follow-up was $26.3 \pm 6.5$ months.

Patient characteristics and number of patients included in each analysis are presented in table 1.

\section{Treatment scheme}

The 610 patients with documented initial dose of BRV were treated with a median daily dose of $100 \mathrm{mg}$ BRV (range 25 to $400 \mathrm{mg}$ ). In 590 patients, BRV was introduced as add-on anticonvulsant therapy with a median of 2 (range 1 to 6 ) other AED. Twenty-two patients were started on a monotherapy with BRV. In three patients concomitant AED were not documented.

A total of 527 patients ( $86 \%$ of the whole sample) had been treated with LEV before. Seventy-three patients (12\% of the whole sample) were LEV naive. There were no statistical differences between both groups regarding $\operatorname{sex}\left(\chi^{2}=0.217, \mathrm{p}=0.64, \mathrm{n}=600\right)$, age $(t(596)=-0.26, \mathrm{p}=0.79)$ or duration of epilepsy $(t(569)=-0.95, \mathrm{p}=0.92)$, both samples fulfilled equality criteria. In 15 patients $(2 \%)$ information on previous LEV therapy was not provided. 
Table 1 Patient characteristics and number of patients included in the analysis

\begin{tabular}{|c|c|c|}
\hline Parameter & $\mathbf{N}$ & $\% *$ \\
\hline Initial daily dose of BRV & 610 & \\
\hline $\begin{array}{l}\text { Information on previous treatment with } \\
\text { LEV }\end{array}$ & 600 & \\
\hline No previous treatment with LEV & 73 & $22 \%$ \\
\hline Previous treatment with LEV & 527 & $88 \%$ \\
\hline Direct switch from LEV to BRV & 237 & $40 \%$ \\
\hline Slow switch from LEV to BRV & 50 & $8 \%$ \\
\hline $\begin{array}{l}\text { LEV discontinued before initiation of } \\
B R V \text { therapy }\end{array}$ & 116 & $19 \%$ \\
\hline $\begin{array}{l}\text { No information how the switch was } \\
\text { performed }\end{array}$ & 124 & $21 \%$ \\
\hline
\end{tabular}

\begin{tabular}{lll} 
Efficacy analysis & 514 & \\
\hline Decreased seizure frequency including & 226 & $44 \%$
\end{tabular}

seizure free

\begin{tabular}{|c|c|c|}
\hline Seizure free & 89 & $17 \%$ \\
\hline Stable seizure frequency & 194 & $38 \%$ \\
\hline Increased seizure frequency & 94 & $18 \%$ \\
\hline $\begin{array}{l}\text { Efficacy analysis in patients on BRV } \\
\text { monotherapy }\end{array}$ & 19 & \\
\hline $\begin{array}{l}\text { Decreased seizure frequency including } \\
\text { seizure free }\end{array}$ & 12 & $63 \%$ \\
\hline Seizure free & 11 & $58 \%$ \\
\hline Stable seizure frequency & 4 & $21 \%$ \\
\hline Increased seizure frequency & 3 & $16 \%$ \\
\hline $\begin{array}{l}\text { Efficacy analysis in patients pretreated } \\
\text { with LEV }\end{array}$ & 441 & \\
\hline $\begin{array}{l}\text { Decreased seizure frequency including } \\
\text { seizure free }\end{array}$ & 184 & $42 \%$ \\
\hline Seizure free & 75 & $17 \%$ \\
\hline Stable seizure frequency & 167 & $38 \%$ \\
\hline Increased seizure frequency & 90 & $20 \%$ \\
\hline Efficacy analysis in LEV naïve patients & 73 & \\
\hline $\begin{array}{l}\text { Decreased seizure frequency including } \\
\text { seizure free }\end{array}$ & 42 & $58 \%$ \\
\hline Seizure free & 14 & $19 \%$ \\
\hline Stable seizure frequency & 27 & $37 \%$ \\
\hline Increased seizure frequency & 4 & $5 \%$ \\
\hline Analysis of tolerability & 615 & \\
\hline Patients with AE on BRV treatment & 169 & $27 \%$ \\
\hline Patients without AE on BRV treatment & 446 & $73 \%$ \\
\hline $\begin{array}{l}\text { Patients with AE on previous LEV } \\
\text { treatment }\end{array}$ & 268 & \\
\hline Improvement after switch to BRV & 168 & $63 \%$ \\
\hline Persistence after switch to BRV & 93 & $35 \%$ \\
\hline Aggravation after switch to BRV & 7 & $2 \%$ \\
\hline Aetiology & 615 & \\
\hline Structural & 312 & $51 \%$ \\
\hline
\end{tabular}

Table 1 Continued

\begin{tabular}{lrr} 
Parameter & N & \multicolumn{1}{c}{ \%* $^{*}$} \\
\hline Cryptogenic & 159 & $26 \%$ \\
Genetic & 39 & $6 \%$ \\
Metabolic & 1 & $0 \%$ \\
Immunological & 6 & $1 \%$ \\
Post-infectious & 4 & $1 \%$ \\
No information & 94 & $15 \%$ \\
\hline
\end{tabular}

*Percent in relation to the number of patients included in this analysis, printed in bold letters, italics: subgroup of the above-mentioned group.

$\mathrm{AE}$, adverse events; BRV, brivaracetam; LEV, levetiracetam.

Of the patients who had been treated with LEV, 237 (45\% of the whole sample) were switched to BRV directly and 50 (9\% of the whole sample) were switched slowly. In 116 patients (22\% of the whole sample) BRV was introduced with a delay after LEV had been discontinued. In the other 124 patients ( $24 \%$ of the whole sample) the switch from LEV to BRV was not further described.

In 116 patients without current LEV therapy, an AED other than LEV was discontinued slowly in exchange for BRV. In one patient, another AED was discontinued simultaneously with the switch from LEV to BRV.

\section{Efficacy}

Of the 514 patients with reliably documented seizures, $226(44 \%)$ had a decreased seizure frequency, 194 (38\%) an unchanged and 94 patients (18\%) an increased seizure frequency.

In the 19 of 22 patients with BRV monotherapy and sufficient information on seizure outcome, $12(63 \%)$ improved, four $(21 \%)$ had no change and three $(16 \%)$ deteriorated.

Of the patients who had been treated with LEV before, changes in seizure frequency after introduction of BRV were reliably documented in 441 patients. One hundred and eighty-four of these patients $(42 \%)$ had a decreased, $167(38 \%)$ a stable and $90(20 \%)$ an increased seizure frequency (figure 1).

Seizures improved in $42(58 \%)$, remained stable in 27 $(37 \%)$ and deteriorated in four $(5 \%)$ of the 73 patients who were LEV naïve.

Complete seizure freedom could be achieved in 89 $(17 \%)$ patients of the total sample, in $75(17 \%)$ of the patients with previous LEV treatment, in $14(19 \%)$ of the LEV naive patients and in $11(58 \%)$ of the patients on BRV monotherapy.

An increase of seizure frequency was significantly more often observed in patients with previous LEV treatment compared with those patients who were LEV naïve $\left(\chi^{2}=9.341, p=0.002, n=514\right)$. A reduction of seizure frequency was significantly more often observed in patients who were LEV naïve compared with patients pretreated with LEV $\left(\chi^{2}=6.355, \mathrm{p}=0.012, \mathrm{n}=514\right)$. These 
Seizure Frequency (SF)

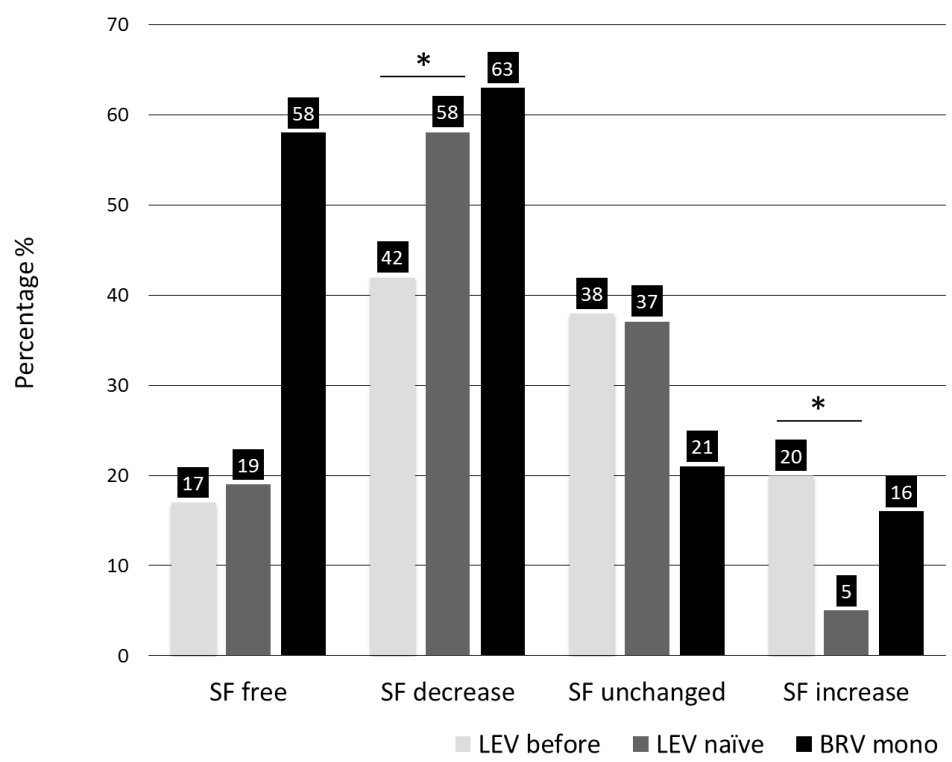

Figure 1 Seizure outcome with brivaracetam(BRV) at latest follow-up for patients who had taken levetiracetam(LEV) before (light grey), LEV naïve patients (dark grey) and patients on BRV monotherapy.

relationships are featured by a small effect size (Cramer's $V=0.13-0.11)$. No significant difference was found between these two groups regarding the proportion of patients with unchanged seizure frequencies $\left(\chi^{2}=0.21\right.$, $\mathrm{p}=0.85, \mathrm{n}=514)$ or patients who achieved seizure freedom $\left(\chi^{2}=0.206, \mathrm{p}=0.65, \mathrm{n}=514\right)$.

Regarding subgroups in aetiology, three groups could be identified for statistical analysis with 312 (51\% of the whole sample) patients suffering from structural, $159(26 \%)$ from cryptogenic and 39 (6\%) from genetic epilepsies. No significant difference was found between these three groups regarding seizure frequency reduction $\left(\chi^{2}=1.434, \mathrm{p}=0.48, \mathrm{n}=510\right)$ or seizure freedom $\left(\chi^{2}=1.549\right.$, $\mathrm{p}=0.46, \mathrm{n}=510)$.

\section{Tolerability}

Overall, 169 of all patients (27\%) reported AE. A significant dose dependent relationship $\left(\chi^{2}=23.35, \mathrm{p}=0.016\right.$, $\mathrm{n}=610$ ) with a small effect size (Cramer's $V=0.19$ ) was observed (table 2).

The most common $\mathrm{AE}$ are summarised in figure 2. Only 2 of 20 patients $(10 \%)$ with BRV monotherapy reported $\mathrm{AE}$, one headache and one loss of appetite. Eleven of the 60 patients with psychiatric comedication $(18 \%)$ reported behavioural AE like depression, aggressiveness, irritability, fear or hallucinations or deterioration of their symptoms, six patients $(10 \%)$ reported non-behavioural AE like dizziness, sleep disturbances, tiredness, weight changes or headache. There were no reports of attempted suicide.

Two hundred and sixty-eight patients $(51 \%$ of the 527 patients on previous LEV treatment) had reported AE while taking LEV, 106 behavioural and 34 nonbehavioural AE. In 128 patients, the LEV-associated AE were not further specified. After switching to BRV, these
AE persisted in $93(35 \%)$ and were aggravated in seven (2\%) of the 268 patients. However, 168 patients (63\%) reported an improvement of previous LEV-associated AE. On the other hand, 60 patients $(23 \%)$ without $\mathrm{AE}$ under treatment with LEV had AE when treated with BRV, 11 (4\%) behavioural and $18(7 \%)$ non-behavioural AE. In 31 $(12 \%)$ the $\mathrm{AE}$ were not further specified.

\section{Retention}

Overall, the proportion of patients still on BRV after a mean follow-up of $26.3 \pm 6.5$ (mean \pm SD) months was $68 \%$. One hundred and ninety-nine of the 615 patients $(32 \%)$

\begin{tabular}{|c|c|c|}
\hline Dose (mg) & Patients without AE & Patients with $\mathrm{AE}$ \\
\hline 25 & 0 & 1 \\
\hline 50 & 60 & 16 \\
\hline 75 & 1 & 4 \\
\hline 80 & 1 & 0 \\
\hline 100 & 161 & 70 \\
\hline 120 & 1 & 0 \\
\hline 125 & 2 & 0 \\
\hline 150 & 37 & 23 \\
\hline 200 & 155 & 53 \\
\hline 250 & 6 & 0 \\
\hline 300 & 15 & 1 \\
\hline 400 & 2 & 1 \\
\hline $\mathrm{N}$ & 441 & 169 \\
\hline
\end{tabular}

$\mathrm{AE}$, adverse event ; BRV, brivaracetam. 


\section{BRV related adverse events}

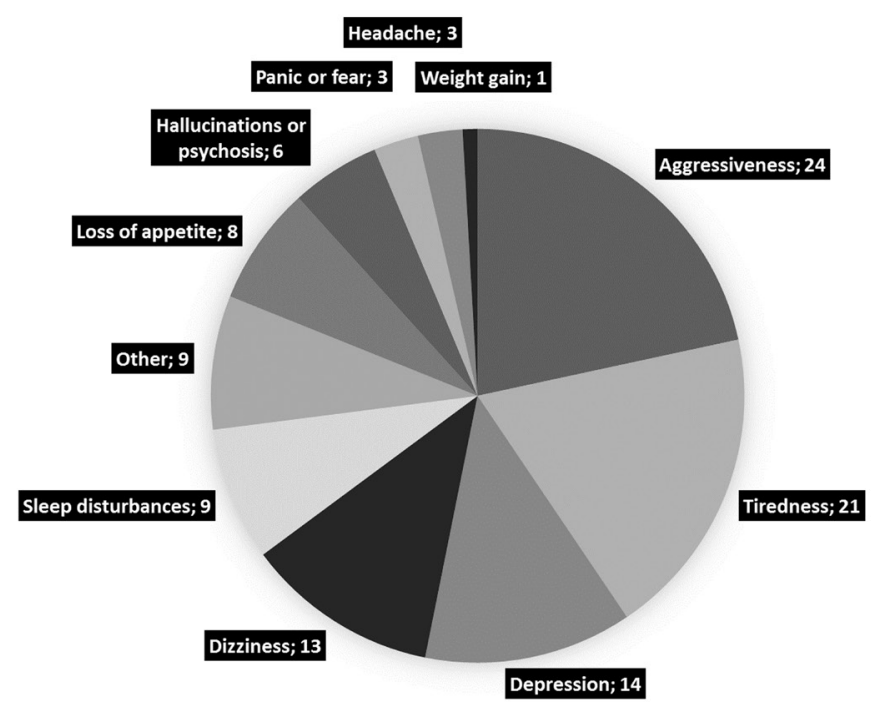

Figure 2 Number of patients with adverse events during therapy with brivaracetam(BRV).

discontinued BRV after $6.8 \pm 5.2($ mean $\pm \mathrm{SD})$ months. The reasons for discontinuation were an increase in seizure frequency in $34(17 \%)$ or lack of improvement of seizure frequency in $43(22 \%)$ of these 199 patients. In 77 patients (39\%), BRV was discontinued due to $\mathrm{AE}$ and in four patients (2\%) to both, lack of efficacy and AE. In 42 of the 199 patients (21\%), the discontinuation had other reasons or the reason was not further specified.

In patients on BRV monotherapy, eight patients (36\%) discontinued BRV after $13.4 \pm 7.6$ (mean \pm SD) months. The proportion of patients still taking BRV after $27.5 \pm 6.4$ $($ mean \pm SD) months was $64 \%$.

\section{DISCUSSION}

The present multicentre study evaluated the effectiveness and tolerability of BRV in daily clinical practice. We could show that BRV is an effective and well tolerated AED and can also be effective in patients who had been treated with LEV before, even though the proportion of patients with reduced seizure frequency was significantly lower in these patients compared with LEV naive patients. Adverse events during treatment with LEV improved in the majority of patients after the switch to BRV. Brivaracetam was also effective and well tolerated when given in monotherapy. The proportion of patients still taking BRV at latest follow-up (mean $\pm \mathrm{SD}=26.3 \pm 6.5$ months) was $68 \%$.

\section{Efficacy}

Overall, our results show improved seizure control in $44 \%$ of the patients. Several phase III studies evaluating the effect of BRV showed $\geq 50 \%$ responder rates of $27.3 \%$ to $32.7 \%$ (BRV $50 \mathrm{mg}$ ), $36.0 \%$ to $38.9 \%$ (BRV $100 \mathrm{mg}$ ) and $37.8 \%$ (BRV $200 \mathrm{mg}) .^{7-9}$ These prospective, placebo-controlled studies had strict inclusion and exclusion criteria like a frequency of at least eight partial onset seizures in the 8 week baseline period and comedication was kept stable. Data were thoroughly collected during predefined follow-up visits and allow for a sophisticated and reliable statistical analysis. On the other hand it remains unclear if results of this special group of patients can be transferred to the heterogeneous patient collective and circumstances of every day clinical practice. The strength of the present study is that patients with all types of epilepsy and any seizure frequency as well as simultaneous changes in other AED were included. Therefore, the present study confirms findings of phase III studies in the clinical setting.

Our results are in line with an earlier multicentre postmarketing study on a smaller group of 262 patients, which showed a responder rate of $40.5 \%$ after a shorter follow-up period of 6 months. ${ }^{10}$

Due to the similar mode of action of LEV and $B R V^{6}$ the benefit of BRV for patients who have been treated with LEV before is of special interest. In the three phase III studies on BRV, concomitant LEV use was either limited to $20 \%^{79}$ of the patient population or patients who had taken LEV within the last 90 days were excluded. ${ }^{8}$ However, these studies point to an effect of BRV even in patients who have previously taken LEV, possibly with a slightly lower responder rate. ${ }^{7-9} 1112$ Our data show that BRV is significantly less effective in patients who have been treated with LEV, but BRV still leads to seizure reduction in $42 \%$ of these patients and there is no significant difference in the proportion of patients who become seizure free compared with patients who are LEV naïve. Even though the efficacy was lower than in LEV naive patients, brivaracetam might therefore be a treatment option even for patients who have been treated with LEV before. 
We also included a subsample of patients with BRV monotherapy. A recently published study reports on two phase III studies which were terminated after a high drop-out rate of about $40 \% .^{13}$ Sufficient data on efficacy and tolerability of BRV monotherapy are therefore lacking. The present results on a limited number of patients with BRV monotherapy show a reduction of seizure frequency in more than half of the patients and a low rate of AE. Further studies on safety and tolerability are therefore warranted.

\section{Tolerability}

A pooled analysis of the three phase III studies reports drug-related $\mathrm{AE}$ of $47.0 \%$ (50 mg/day), 39.9\% (100 $\mathrm{mg} /$ day) and $43.6 \%$ (200 mg/day). ${ }^{6}$ Our results support these findings and even show a slightly lower rate of $\mathrm{AE}$ in the clinical setting. This lower number might however be due to the retrospective study design and lack of systematic documentation of adverse events in the present study and should therefore be interpreted with caution. Interestingly, the number of behavioural $\mathrm{AE}$ and deterioration of psychiatric condition was low $(18 \%)$ in patients with psychiatric medication, an indicator for psychiatric comorbidities. Brivaracetam therefore appears to be a treatment option even in those patients seemingly at higher risk for psychiatric adverse events.

Including all patients who had been treated with LEV before and experienced AEs we could show an improvement of LEV-associated AEs during the treatment with BRV in $63 \%$ of 268 patients. In a pooled analysis of patients who switched to BRV due to behavioural AE during treatment with LEV, 27 out of 29 patients (93\%) had a clinically meaningful reduction in behavioural $\mathrm{AE}$ as rated by the physician. ${ }^{12}$ The small number of 29 patients, strict inclusion criteria allowing only an immediate switch and the stable comedication in this earlier study might explain the higher percentage of patients in whom AE improved after the switch to BRV. The improvement of LEV-associated $\mathrm{AE}$ in $62 \%$ of patients in the present study is however in line with results of another study, where 44 out of 57 patients $(77 \%)$ had a clinically meaningful reduction of overall $\mathrm{AE}$ and 24 out of $36(67 \%)$ of behavioural AE after switching from LEV to BRV. ${ }^{14}$ A switch to BRV might therefore be a treatment option for patients with LEV-associated AE.

\section{CONCUSIONS AND LIMITATIONS}

In this multicentre study on postmarketing experiences with BRV in clinical settings, BRV seemed to be an easy to use, easy to switch and well tolerated new antiepileptic drug with more than $60 \%$ of patients still taking BRV even after a follow-up period of more than 2 years. Brivaracetam also appears to be effective and well tolerated when given as monotherapy and might even be a treatment option for patients with psychiatric comorbidities or those who failed LEV treatment. We observed a weak relationship between BRV dose and adverse events. However, this result should be interpreted with caution as sample sizes in some dose subgroups were small.

This study is a multicentre retrospective study. Data were collected by questionnaires that were answered by the reporting neurologist using patient charts. Therefore, limitations of this study include missing data, data that were not collected in a systematic way, variable follow-up intervals and changes in comedication. On the other hand, the group of patients is representative for the group of patients treated with BRV in the clinical setting as all patients treated with BRV and a follow-up period of at least 3 months were included in the 10 participating centres. Another limitation of this retrospective study is the lack of a control-group receiving placebo. Due to these limitations of the study results are mainly presented in a descriptive manner to avoid overinterpretation of the results.

\section{Author affiliations}

${ }^{1}$ Epilepsy Center Hessen, Department of Neurology, Philipps-University Marburg, Marburg, Germany

${ }^{2}$ Epilepsy Center Frankfurt Rhine-Main, Department of Neurology, Center of Neurology and Neurosurgery, University Hospital, Goethe-University Frankfurt, Frankfurt am Main, Germany

${ }^{3}$ Center for Personalized Translational Epilepsy Research (CePTER)

${ }^{4}$ Department of Child Neurology, Goethe-University Frankfurt am Main, Frankfurt am Main, Germany

${ }^{5}$ Epilepsy Center Greifswald and Department of Neurology, Ernst-Moritz-ArndtUniversity, Greifswald, Germany

${ }^{6}$ Epilepsy Center, Faculty of Medicine, University of Freiburg, Freiburg, Germany ${ }^{7}$ Epilepsy Center Erlangen, Department of Neurology, University of Erlangen, Erlangen, Germany

${ }^{8}$ Epilepsy Center Tuebingen, Department of Neurology, University Hospital Tuebingen, Tuebingen, Germany

${ }^{9}$ Epilepsy Center Mainz, Department of Neurology, Johannes Gutenberg-University Mainz, Mainz, Germany

${ }^{10}$ Department of Neurology, University of Münster, Münster, Germany

${ }^{11}$ Sächsisches Epilepsiezentrum Radeberg gGmbH, Kleinwachau, Germany

${ }^{12}$ Hospital Center of Luxembourg, Luxembourg, Luxembourg

Acknowledgements We thank Isabel Steinig for her help with preparing the data from Frankfurt.

Contributors KM: analysis and interpretation of the data, writing of the manuscript. PMM: acquisition of data, analysis and interpretation of the data. FR: acquisition of data, revision of the manuscript. SSB: acquisition of data, revision of the manuscript. LMW: acquisition of data, revision of the manuscript. FZ: acquisition of data, revision of the manuscript. II: acquisition of data, revision of the manuscript. SF: acquisition of data, revision of the manuscript. FvP: acquisition of data, revision of the manuscript. RK: acquisition of data, revision of the manuscript. $\mathrm{MH}$ : acquisition of data, revision of the manuscript. TM: acquisition of data, revision of the manuscript. JM: acquisition of data, revision of the manuscript. YW: acquisition of data, revision of the manuscript. LL: acquisition of data, revision of the manuscript. MC: acquisition of data, revision of the manuscript. SB: acquisition of data, revision of the manuscript. AS: acquisition of data, revision of the manuscript. SK: study concept and design, analysis and interpretation of the data, revision of the manuscript.

Funding The authors have not declared a specific grant for this research from any funding agency in the public, commercial or not-for-profit sectors.

Competing interests $\mathrm{KM}$ has received honoraria as advisory board member from Eisai and UCB. FR has received personal fees from Bayer-Vital, Cerbomed, Eisai, Hexal and Sandoz, personal fees and support for continuing medical education activities from Desitin, Novartis, Shire and UCB Pharma and grants from DFG (German Research Foundation) and the European Union. SS-B reports personal 
fees from UCB, Eisai, Desitin Pharma, LivaNova, Bial, Novartis and Zogenix outside of the submitted work. FVP reports industry-funded travel with support of Desitin Arzneimittel, UCB Pharma, Eisai Pharma and BIAL, obtained honoraria for speaking engagements from Desitin Arzneimittel, UCB Pharma, Eisai Pharma and BIAL and was part of a speaker's bureau of Desitin Arzneimittel, UCB Pharma and BIAL. RK reports industry-funded travel with support of Eisai Pharma and Desitin Arzneimittel. MH has received honoraria for lectures from pharmaceutical companies (Eisai, UCB) and research support for participation in clinical trials and registries from Medtronic, Cyberonics and Precisis. TM has received travel expenses for attending a meeting from UCB Pharma. YW received travel grants and honoraries for lectures from UCB Pharma, BIAL, Eisai, Bayer and Novartis, which were not related to the publication. LL has received lecture honoraria from Eisai. MC has received travel expenses for attending meetings from Desitin and Zogenix. AS reports personal fees and/or grants from Desitin Arzneimittel, Eisai, GW Pharma, LivaNova, Medtronic, Sage Therapeutics, UCB Pharma and Zogenix. SK reports honoraria for speaking engagements from Desitin and UCB as well as educational grants from AD Tech, Desitin Arzneimittel, Eisai, GW, Medtronic, Novartis, Siemens and UCB.

Patient consent for publication Not required.

Ethics approval The study was approved by local ethic committees.

Provenance and peer review Not commissioned; externally peer reviewed.

Data availability statement Data are available upon reasonable request.

Open access This is an open access article distributed in accordance with the Creative Commons Attribution Non Commercial (CC BY-NC 4.0) license, which permits others to distribute, remix, adapt, build upon this work non-commercially, and license their derivative works on different terms, provided the original work is properly cited, appropriate credit is given, any changes made indicated, and the use is non-commercial. See: http://creativecommons.org/licenses/by-nc/4.0/.

\section{ORCID iDs}

Katja Menzler http://orcid.org/0000-0001-8289-480X

Susanne Schubert-Bast http://orcid.org/0000-0003-1545-7364

Laurent Maximilian Willems http://orcid.org/0000-0001-8226-1674

\section{REFERENCES}

1 Hirtz D, Thurman DJ, Gwinn-Hardy K, et al. How common are the "common" neurologic disorders? Neurology 2007;68:326-37.
2 Kwan P, Brodie MJ. Early identification of refractory epilepsy. N Engl J Med Overseas Ed 2000;342:314-9.

3 Wilby J, Kainth A, Hawkins N, et al. Clinical effectiveness, tolerability and cost-effectiveness of newer drugs for epilepsy in adults: a systematic review and economic evaluation. Health Technol Assess 2005;9:1-157.

4 Chung S, Wang N, Hank N. Comparative retention rates and long-term tolerability of new antiepileptic drugs. Seizure 2007;16:296-304

5 Strzelczyk A, Klein KM, Willems LM, et al. Brivaracetam in the treatment of focal and idiopathic generalized epilepsies and of status epilepticus. Expert Rev Clin Pharmacol 2016;9:637-45.

6. Ben-Menachem E, Mameniškienė R, Quarato PP, et al. Efficacy and safety of brivaracetam for partial-onset seizures in 3 pooled clinical studies. Neurology 2016;87:314-23.

7 Ryvlin P, Werhahn KJ, Blaszczyk B, et al. Adjunctive brivaracetam in adults with uncontrolled focal epilepsy: results from a double-blind, randomized, placebo-controlled trial. Epilepsia 2014;55:47-56.

8 Klein P, Schiemann J, Sperling MR, et al. A randomized, doubleblind, placebo-controlled, multicenter, parallel-group study to evaluate the efficacy and safety of adjunctive brivaracetam in adult patients with uncontrolled partial-onset seizures. Epilepsia 2015;56:1890-8.

9 Biton V, Berkovic SF, Abou-Khalil B, et al. Brivaracetam as adjunctive treatment for uncontrolled partial epilepsy in adults: a phase III randomized, double-blind, placebo-controlled trial. Epilepsia 2014;55:57-66.

10 Steinig I, von Podewils F, Möddel G, et al. Postmarketing experience with brivaracetam in the treatment of epilepsies: a multicenter cohort study from Germany. Epilepsia 2017;58:1208-16.

11 Asadi-Pooya AA, Sperling MR, Chung S, et al. Efficacy and tolerability of adjunctive brivaracetam in patients with prior antiepileptic drug exposure: a post-hoc study. Epilepsy Res 2017;131:70-5.

12 Yates SL, Fakhoury T, Liang W, et al. An open-label, prospective, exploratory study of patients with epilepsy switching from levetiracetam to brivaracetam. Epilepsy Behav 2015;52:165-8.

13 Arnold S, Badalamenti V, Diaz A, et al. Conversion to brivaracetam monotherapy for the treatment of patients with focal seizures: two double-blind, randomized, multicenter, historical control, phase III studies. Epilepsy Res 2018;141:73-82.

14 Zahnert F, Krause K, Immisch I, et al. Brivaracetam in the treatment of patients with Epilepsy-First clinical experiences. Front Neurol 2018;9:38. 